P023

A MIXED-METHODS ANALYSIS OF A PAY-IT-FORWARD GONORRHEA/CHLAMYDIA TESTING PROGRAM AMONG MEN WHO HAVE SEX WITH MEN IN CHINA

${ }^{1}$ Katherine Li, ${ }^{2}$ Fan Yang, ${ }^{3}$ Wenting Huang*, ${ }^{2}$ Yang Zhao, ${ }^{4}$ Dan Wu, ${ }^{5}$ Weiming Tang, ${ }^{6}$ Feng Wu, ${ }^{7}$ Ligang Yang, ${ }^{8}$ Tiange Zhang, ${ }^{4}$ Kate Muessig, ${ }^{5}$ Joseph Tucker. ${ }^{1}$ Weill Cornell Medical College, New York, USA; 'University of North Carolina Project - China, Guangzhou, China; ${ }^{3}$ University of North Carolina Project-China, Guangzhou, China; ${ }^{4}$ University of North Carolina at Chapel Hill, Chapel Hill, USA; ${ }^{5}$ UNC Project-China, Guangzhou, China; ${ }^{6}$ Sun Yat-sen University, Department of Sociology, Guangzhou, China; ${ }^{7}$ Dermatology Hospital, Southern Medical University, Guangzhou, China; ${ }^{8}$ Stritch School of Medicine, Loyola University, Maywood, USA

10.1136/sextrans-2019-sti.232

Background Neisseria gonorrhoeae and Chlamydia trachomatis are common among Chinese men who have sex with men (MSM), but testing rates are low. We developed a pay-it-forward program, where each man receives a free gonorrhea/ chlamydia test and can then choose to donate toward testing for future participants. This study aims to investigate the facilitators of increased gonorrhea/chlamydia testing among MSM in the pay-it-forward program.

Methods We collected survey data on socio-demographics and perceived benefits of the pay-it-forward program among men offered the pay-it-forward intervention as part of a quasiexperimental study in Guangzhou, China. We analyzed testing uptake using multivariable logistic regression. We also conducted twelve semi-structured interviews with men who received testing eliciting their perceptions of the program. Interview data were coded to identify themes.

Results 204 MSM were offered pay-it-forward and 109 (54\%) received gonorrhea/chlamydia testing. Across survey and interview data, the main facilitators of testing were (1) lower cost and (2) care for MSM community. First, 48/204 (24\%) participants selected discounted testing as a benefit of pay-it-forward, and receiving testing was not associated with income. In interviews, the majority of participants felt that standard hospital-based testing was prohibitively expensive, especially for students (150 RMB, USD \$21.50); pay-it-forward made testing affordable for them. Second, 141/204 (70\%) men selected 'more MSM can get tested' or 'I can help someone else' as benefits of pay-it-forward, and selecting at least one of these options was associated with getting tested $(\mathrm{aOR}=2.59,95 \%$ $\mathrm{CI}=1.31-5.15)$. In interviews, some men expressed desire to contribute to the MSM community; others felt that encouraging others to test could also eventually benefit themselves.

Conclusion This pay-it-forward program may increase MSM gonorrhea/chlamydia testing rates by reducing barriers due to cost and mobilizing community altruism. This model may be useful for other settings and sexual health services.

Disclosure No significant relationships.

\section{P024 TARGETED POINT-OF-CARE TESTING COMPARED TO SYNDROMIC MANAGEMENT OF UROGENITAL INFECTIONS IN RWANDAN WOMEN}

${ }^{1}$ Janneke Van De Wijgert ${ }^{*},{ }^{2}$ Marijn Verwijs, ${ }^{3}$ Stephen Agaba, ${ }^{3}$ Jean Claude Sumanyi, ${ }^{3}$ Marie Michelle Umulisa, ${ }^{3}$ Lambert Mwambarangwe, ${ }^{3}$ Viateur Musengamana, ${ }^{3}$ Mireille Uwineza, ${ }^{4}$ Vicky Cuylaerts, ${ }^{4}$ Tania Crucitti, ${ }^{4}$ Vicky Jespers. ${ }^{1}$ University Medical Center Utrecht, Julius Center for Health Sciences and Primary Care, Utrecht, Netherlands; ${ }^{2}$ University of Liverpool, Institute of Infection and Global Health, Liverpool, UK; ${ }^{3}$ Rinda Ubuzima, Kigali, Rwanda; ${ }^{4}$ Institute of Tropical Medicine, Antwerp, Belgium

10.1136/sextrans-2019-sti.233
Background Sexually transmitted and urogenital infections are typically managed by World Health Organisation (WHO)-recommended syndromic algorithms in resource-poor countries. Vaginal discharge (VDS) and lower abdominal pain (LAP) algorithms in women perform poorly. The main aim of the WISH study was to compare the performances of VDS/LAP algorithms incorporating point-of-care tests (POCTs), and of WHO syndromic algorithms, with gold standard test results.

Methods At-risk Rwandan women $(\mathrm{N}=705)$ underwent POCTs for bacterial vaginosis ( $\mathrm{BV}$; vaginal $\mathrm{pH} \geq 5 \cdot 0)$ and Trichomonas vaginalis (TV; OSOM) regardless of symptom-reporting. Women with a positive risk score were POC-tested for Chlamydia trachomatis and Neisseria gonorrhoeae (CT/NG; GeneXpert). Vulvovaginal candidiasis (VVC) was treated presumptively. Nucleic acid amplification tests (NAATs) were done for $\mathrm{CT} / \mathrm{NG}, \mathrm{TV}, \mathrm{BV}$, and $\mathrm{VVC}$ on everyone and were used as gold standards.

Results NAAT-based prevalences were: 60/705 (8.5\%) CT, 50/ $705(7 \cdot 1 \%)$ NG, 111/690 (16.1\%) TV, 125/690 (18.1\%) BV, and $59 / 690(8 \cdot 6 \%)$ VVC. Infection-specific sensitivities of the WHO VDS/LAP algorithms ranged from $58 \cdot 3-74 \cdot 6 \%$, and specificities from $44 \cdot 7-50 \cdot 6 \%$. WISH POCT-based algorithms had good sensitivity $(68 \cdot 5-76 \cdot 0 \%)$ and specificity $(97 \cdot 4-100 \%)$ for CT, NG, and TV but low specificity for BV $(41 \cdot 2 \%$; sensitivity $95 \cdot 2 \%)$, and modest sensitivity (64.4\%) and specificity (69.4\%) for VVC. Sensitivity (73.6\%) and specificity (100\%) for BV improves by screening all women for vaginal $\mathrm{pH}$, and confirmatory testing of those with $\mathrm{pH} \geq 5 \cdot 5 \quad(\mathrm{n}=275)$. Speculum/bimanual examinations by a physician had limited added value (except in the case of LAP), and partner notification was suboptimal. Staff and participants considered POC testing feasible and acceptable.

Conclusion POC testing for urogenital infections in women improves performance and is feasible in resource-poor settings. The WHO VDS/LAP algorithms should therefore recommend POC testing whenever feasible. However, programmes would benefit from more affordable combined CT/NG POCTs, and POCTs combining BV, TV, and VVC diagnoses. Additional studies in other populations, including low prevalence populations, are warranted.

Disclosure No significant relationships.

\section{P025 AUTOMATION AND OPTIMIZATION STRATEGIES IN A MOLECULAR LAB RESULT IN AN IMPROVED WORKFLOW FOR STI SURVEILLANCE STUDIES}

${ }^{1}$ Carsten Tiemann*, ${ }^{2}$ Gyde Steffen, ${ }^{3}$ Ann-Kathrin Schuppe, ${ }^{2}$ Viviane Bremer, ${ }^{4}$ Klaus Jansen. ${ }^{1}$ Krone Laboratory/LABCON-OWL, Molecular Diagnostics, Bad Salzuflen, Germany; ${ }^{2}$ Robert Koch Institute, Berlin, Germany; ${ }^{3}$ Krone Laboratory, Bad Salzuflen, Germany; ${ }^{4}$ Robert Koch Institute, Infectious Disease Epidemiology, Berlin, Germany

10.1136/sextrans-2019-sti.234

Background Clinical types of specimen as well as rising sample numbers during a STI studies, pose a challenge to the capability of the lab. Workflow optimization and automation strategies are crucial to enable high throughput investigations with a maximum of efficiency if diagnostic data have to be combined with additional information content.

Methods In our molecular laboratory we have established an optimized the sample management, automated extraction and detection protocols using the Panther ${ }^{\circledR}$ instrument, bidirectional order entry and reporting setting followed by a digital archiving and data management procedure. The workflow 